zu Antibiotika und chirurgischen Eingriffen bis heute dringend gesucht. Francis und Team haben daher getestet, ob eine Kortisonstoßtherapie den Heilungsprozess beschleunigen könnte.

Antibiotika, topische intranasale Steroide, Antihistaminika, Mukolytika - die verschiedenen Ansätze zur Behandlung einer Otitis media mit Paukenerguss im Kleinkindalter sind sehr vielfältig, haben sich aber in vielen Studien als ineffektiv erwiesen. Da der begleitende Hörverlust die sprachliche Entwicklung der betroffenen Patienten gefährden kann, bleibt bis heute der Bedarf an wirksamen Behandlungsansätzen.

Weil zudem die chirurgische Anlage eines Paukenröhrchen von den Kindern als unangenehm empfunden werden kann und mit relativ hohen Behandlungskosten einhergeht, scheint eine Stoßtherapie mit dem Kortikosteroid Prednisolon eine kostengünstige und leicht durchzuführende Alternative zu sein. Aufgrund einer eher schwachen Studienlage zum Thema haben Francis und Kollegen nun mit der Oral Steroids for Resolution of Otitis Media with Effusion in Children (OSTRICH) eine doppelblinde und Placebo-kontrollierte, randomisierte Studie durchgeführt.

Die Untersuchung fand dabei zwischen März 2014 und April 2016 statt und schloss 20 ausgewiesene Zentren aus England und Wales mit ein. Geeignete Patienten erfüllten die folgenden Einschlusskriterien:

\section{Pädiatrie: Prednisolon bei Otitis media nicht besser als Placebo?}

Francis NA et al. Oral steroids for resolution of otitis media with effusion in children (OSTRICH): a double-blinded, placebo-controlled randomised trial. Lancet 2018; doi:10.1016/S01406736(18)31490-9

Eine Mittelohrentzündung mit Paukenerguss gilt bei Kleinkindern als häufige Diagnose und kann durch den begleitenden Hörverlust die Sprachentwicklung negativ beeinflussen. Da sich viele Therapiestrategien als unwirksam erwiesen haben, werden Alternativen
- Alter zwischen 2 und 8 Jahren

- Otitis media mit Paukenerguss für min. 3 Monate

- nachgewiesener symptomatischer Hörverlust beider Ohren

Zu den wichtigsten Ausschlusskriterien zählten die Forscher angeborene genetische Erkrankungen oder schwere Komorbiditäten. Nach Rekrutierung und Randomisierung teilten die Forscher jeden Patienten einer von zwei Studiengruppen zu:

- Interventionsgruppe (IG): Prednisolon 20 mg (bei Kindern im Alter von 2 - 5 Jahren) oder $30 \mathrm{mg}$ (bei Kindern im Alter von 6 - 8 Jahren) 1-mal tgl. für 7 Tage

- Kontrollgruppe (KG): Placebo 1-mal tgl. für 7 Tage 


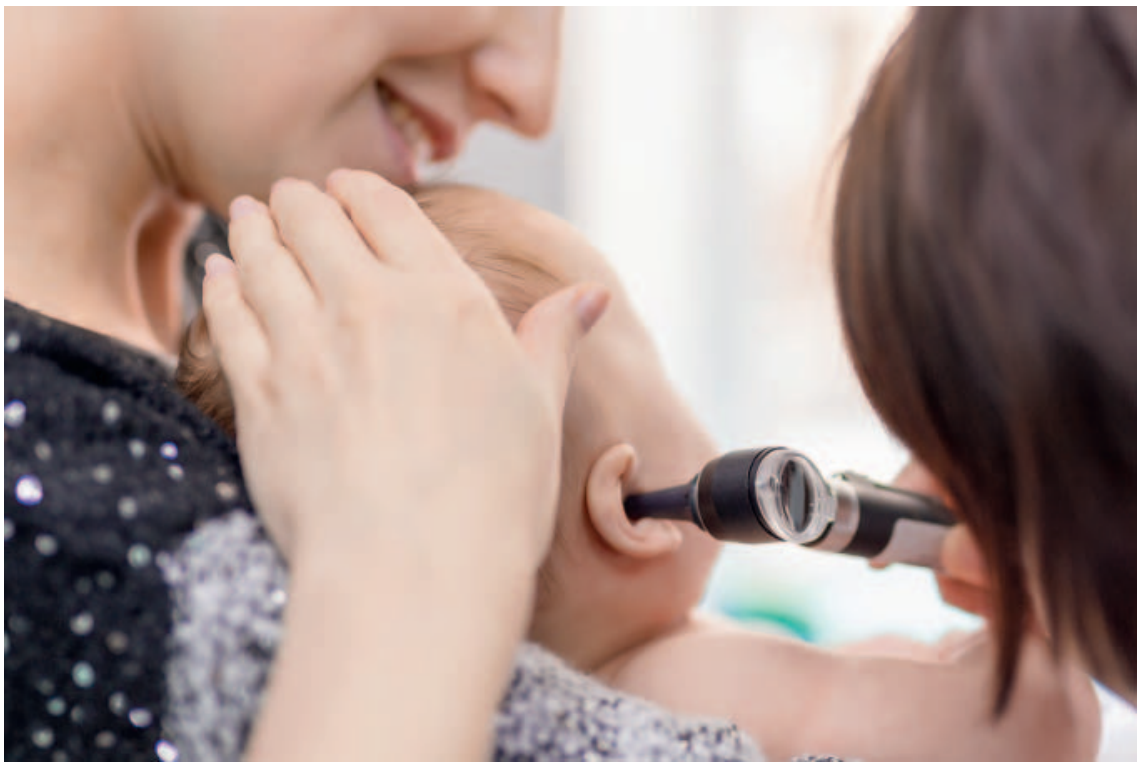

In der Pädiatrie ist die Diagnose einer Mittelohrentzündung mit Paukenerguss häufig: Der begleitende Hörverlust kann dabei die Sprachentwicklung negativ beeinflussen. Francis et al. untersuchten daher, ob eine Kortisonstoßtherapie den Heilungsprozess beschleunigen könnte. Symbolbild; Bildnachweis: Adobe Stock/Kirill Gorlov

Als primären klinischen Endpunkt definierten die Studienautoren ein akzeptables Hörvermögen 5 Wochen nach Randomisierung. Zu den sekundären Endpunkten zählten unter anderem die Erholung des Hörvermögens nach 6 und nach 12 Monaten, das Ergebnis der Tympanometrie und otoskopischer Untersuchungen, der funktionelle Gesundheitsstatus sowie die gesundheitsbezogene Lebensqualität. Darüber hinaus betrachteten die Forscher die kurzzeitige und längerfristige Kosteneffektivität.

\section{Hohe Spontanheilungsrate}

Von 1018 geeigneten Kindern konnten 389 (38\%) randomisiert werden. 380 Kinder nahmen schließlich bis zum Schluss an der Studie teil, 193 von ihnen in der Interventionsgruppe und 187 in der Kontrollgruppe. Die kleinen Patienten waren durchschnittlich 5,3 (IG) bzw. 5,8 (KG) Jahre alt, 57 bzw. $55 \%$ von ihnen männlich.

73 Kinder (40\%) der Interventionsgruppe und 59 (33\%) der Kontrollgruppe erreichten den primären klinischen Endpunkt und zeigten 5 Wochen nach Randomisierung eine deutliche Erholung des zunächst eingeschränkten Hörvermögens. Dieser Unterschied war dabei statistisch nicht sig- nifikant, die spontane Heilungsrate wurde von den Forschern als sehr hoch eingestuft.

Auch für die sekundären klinischen Endpunkte konnten die Forscher keine deutlichen und robusten Gruppenunterschiede feststellen. Art und Anzahl von registrierten Nebenwirkungen waren für beide Studiengruppen vergleichbar. Im Gegensatz zur Behandlung mit Placebo schnitt eine Therapie mit Prednisolon hinsichtlich Kosteneffektivität etwas schlechter ab.

\section{FAZIT}

Bei Kindern im Alter zwischen 4 und 11 Jahren mit Otitis media und Paukenerguss für mindestens drei Monate konnte eine Stoßtherapie mit Prednisolon die Erholung des Hörvermögens nicht wesentlich beschleunigen. Aufgrund der sehr hohen Spontanheilungsrate halten die Autorinnen/Autoren das sogenannte „Watchful Waiting“ für eine sinnvolle, nebenwirkungsarme und auch kosteneffektive Behandlungsstrategie.

Dipl.-Psych. Annika Simon, Hannover 